\title{
¿Sirve el tratamiento para rehabilitar a los delincuentes sexuales? ${ }^{1}$
}

\author{
Santiago Redondo Illescas ${ }^{2}$
}

\author{
Departamento de Personalidad, Evaluación y Tratamiento Psicológico \\ Facultad de Psicología, Universidad de Barcelona \\ P. de la Vall d'Hebron 171 \\ 08035-Barcelona
}

\section{RESUMEN}

En este trabajo se describe y evalúa el tratamiento psicológico que se aplica en la prisión de Brians (en Barcelona, España) con los agresores sexuales. Para poder evaluar la eficacia del tratamiento se han constituido dos grupos equivalentes (uno de tratamiento y otro de control). El grupo de tratamiento está integrado por 49 sujetos que recibieron, de manera completa, el tratamiento psicológico previsto. Por su parte, el grupo de control lo integran 74 agresores sexuales que no habían recibido tratamiento. Para seleccionar los sujetos integrantes de los grupos se tomaron en cuenta diversos factores de riesgo, con la finalidad de asegurar la equivalencia y posibilidad de comparación de los grupos. Los resultados muestran con claridad que este programa cognitivo-conductal tiene una gran potencia rehabilitadora con los delincuentes sexuales. Pese a todo, diversos factores estáticos de riesgo, tales como la edad de los sujetos, la duración de sus carreras delictivas, el número de delitos que habían cometido anteriormente, etc., condicionaron el grado de eficacia del programa. Por último, se debate el papel que, aparte de los factores personales de riesgo que son objetivo del tratamiento, pueden jugar distintos factores sociales, como facilitadores o entorpecedores de la rehabilitación de los delincuentes sexuales.

\footnotetext{
${ }^{1}$ Este trabajo se ha desarrollado en el marco del Proyecto de Investigación Coordinado (SEJ2005-09170C04-01) sobre "Delincuencia violenta: Programas de prevención, tratamiento y predicción" (2005-2008) financiado por el Ministerio de Educación.

${ }^{2}$ Mi gratitud a mis buenos colegas y amigos Joan Carles Navarro, Eulalia Luque y Marian Martínez, sin cuyo trabajo esforzado y ayuda hubiera sido imposible el desarrollo de la investigación empírica presentada en este informe.
}

Revista Española de Investigación Criminológica Artículo 6, Número 4 (2006) $\quad$ www.criminología.net 


\begin{abstract}
This paper describes and assesses the psychological treatment applied at the Brians prison (Barcelona, Spain) to sex offenders. In order to assess the treatment effectiveness, two equivalent groups (treatment and control) are selected. The treatment group is formed of 49 subjects that had received the whole treatment program, and the control group of 74 subjects that hadn't received treatment. These groups are been selected taking into account different risk factors in order to guarantee the comparability between the groups. The results show that this cognitive-behavioural program has a great rehabilitative potency with sex offenders. Nevertheless, the effectiveness of the program is mediated by some static risk factors (as the subject's age, the duration of their criminal career, the number or previous committed crimes, etc.). Finally, it is discussed that, apart from personal factors that can be changed by the application of treatment, other social factors can facilitate or difficult the rehabilitation of sexual offenders.
\end{abstract}

\title{
1. Riesgo de reincidencia y tratamiento de los agresores sexuales
}

Los agresores sexuales constituyen un reto importante para la criminología aplicada debido a la complejidad de factores de riesgo que confluyen en ellos. Según nuestros conocimientos actuales, los agresores sexuales suelen presentar carencias de tres tipos diferentes aunque interrelacionados: en su comportamiento y preferencias sexuales (lo que resulta obvio), en su conducta social más amplia, y en sus cogniciones (“distorsiones cognitivas”) (Berlin, 2000; Brown, 2005; Echeburúa y Guerricaechevarría, 2000; Marshall, 2001; Redondo, 2002). Así pues, todas estas dificultades deberán ser consideradas en el diseño y aplicación de un programa de tratamiento.

Puede afirmarse que en la actualidad se dispone de buenas estrategias psicológicas para el tratamiento de los agresores sexuales, a un nivel de desarrollo técnico comparable al de otros campos de la intervención psicológica (Budrionis y Jongsma, 2003). Sin embargo, desde la perspectiva de la utilización práctica de tales técnicas, la aplicación de tratamientos a los delincuentes sexuales es una actividad limitada a unos pocos países desarrollados y, dentro de ellos, a unos cuantos programas en prisiones, y esporádicamente en la propia comunidad. Ello significa que la proporción de agresores sexuales que recibe tratamiento es muy pequeña en relación con el número de agresores sexuales identificados $y$, normalmente, encarcelados. Pese a todo, los poderes públicos son cada vez más

Revista Española de Investigación Criminológica

Artículo 6, Número 4 (2006) $\quad$ www.criminología.net

ISSN: 1696-9219 
conscientes de la necesidad de aplicar tratamientos especializados a los delincuentes sexuales y por ello, en los países norteamericanos y europeos, se van introduciendo paulatinamente nuevos programas. Tales programas suelen tener las siguientes características generales:

- Suelen ser programas intensivos de larga duración.

- Incluyen técnicas dirigidas específicamente a las tres áreas problemáticas mencionadas: comportamiento sexual desviado, distorsiones cognitivas y funcionamiento social del sujeto.

- A veces se utilizan, en el contexto global del programa, agentes químicos inhibidores del impulso sexual. Se han utilizado tres medicaciones reductoras del impulso sexual masculino: el acetato de ciproterona, el acetato de medroxiprogesterona y, más modernamente, el agonista análogo de la hormona liberadora de la gonadotropina (GnRH) (Greenberg y Bradford, 1997; Marshall y Redondo, 2002; Rösler y Witztum, 2000).

- En la aplicación suelen intervenir diversos terapeutas (con frecuencia hombre y mujer) que entrenan a los agresores sexuales en habilidades sociales específicas con un doble propósito: 1) que aprendan a inhibir las conductas delictivas, y 2) que aprendan las habilidades de comunicación necesarias para establecer relaciones sexuales adultas y consentidas.

- En general, el tratamiento suele tener carácter voluntario, aunque la eventual participación del sujeto en el mismo suele ser recompensada con beneficios penales y penitenciarios (permisos de salida al exterior, mejora del régimen de vida en prisión, o concesión futura de la libertad condicional).

De acuerdo con diversos estudios evaluativos, la reincidencia de los agresores sexuales es, como grupo, baja, y se estima de en torno al 20\% (Lösel, 2002; Prentky, 2003; Quinsey et al., 1995). (El promedio general de la reincidencia de los delincuentes -no específicamente sexuales- es de alrededor del 50\%). No obstante, la distribución de la reincidencia es muy heterogénea y oscila entre un número grande de casos de un solo delito conocido (y, por tanto, no reincidentes) y, 
en el extremo opuesto, unos pocos agresores sexuales seriales, que cometen decenas de delitos a lo largo de sus carreras criminales.

En cada caso, el riesgo de reincidencia -y también las posibilidades del tratamiento- van a depender de la tipología de agresor de que se trate y, específicamente, de los factores de riesgo que confluyan en cada sujeto. La investigación más moderna en psicología criminal ha puesto de relieve la existencia tanto de factores de riesgo -aumentan el riesgo- como de factores de protección o resistencia -protegen al individuo, disminuyendo el riesgo de conducta delictiva(Garrido, 2005). A su vez, unos y otros factores se han categorizado como factores estáticos (o inmodificables) y dinámicos (o modificables mediante intervenciones) (Andrews y Bonta, 2003; Andrews, Zinder, Hoge, Bonta, Gendreau y Cullen, 1990; Gendreau, Little y Goggin, 1996). Los factores de riesgo estáticos suelen ser factores inherentes al sujeto o a su pasado y, por ello, de difícil o imposible alteración, mientras que los factores de riesgo dinámicos consisten en hábitos, valores, cogniciones, bajo estatus académico y social, bajo autocontrol, conflictos interpersonales, etc., todos los cuales pueden modificarse en cierto grado mediante intervenciones apropiadas.

Existen predictores o factores de riesgo específicos de reincidencia sexual, que también pueden ser divididos en predictores estáticos y dinámicos. Los principales son los siguientes (Andrews y Bonta, 2003; Berlin, 2000; Gallagher et al., 1999; Groth, 1979; Quinsey et al., 1995; Maletzky, 1991; Marshall, 2001; Marshall y Barbaree, 1989, 1990; Marshall y Redondo, 2002; Vandiver, 2006) (tabla 1): 
Tabla 1. Predictores de riesgo de reincidencia sexual

\begin{tabular}{|c|c|}
\hline Predictores estáticos & Predictores dinámicos \\
\hline 1.[Factor genérico: menor edad] & 1.Negación $\quad$ o \\
\hline 2.Mayor número de delitos sexuales previos & persistente del delito \\
\hline 3.Mayor número de delitos previos en general & 2.Baja/nula motivación para seguir \\
\hline 4.Versatilidad delictiva (no especialización sexual) & un tratamiento \\
\hline 5.Violencia en la realización de los delitos previos & 3.Baja competencia interpersonal y, \\
\hline $\begin{array}{l}\text { 6.Escalamiento en la gravedad de los delitos (violencia, } \\
\text { víctimas menores) }\end{array}$ & $\begin{array}{ll}\text { en general, factores de } \\
\text { necesidad } & \begin{array}{l}\text { criminogénica } \\
\text { crimata }\end{array}\end{array}$ \\
\hline 7.Víctima & (cogniciones, valores y hábitos \\
\hline 8.Tip & delictivos, $\quad$ amigos \\
\hline -Femeninas (.17) & dependencia \\
\hline -Masculinas $\quad(\mathbf{2 0})$ & drog \\
\hline -Infantiles (.22) & 4.Alta excitación ante estímulos \\
\hline -Adultas (.13) & sexuales desviados y baja \\
\hline $\begin{array}{l}\text { 9.El delito no ha sido resultado de algún estresor } \\
\text { específico circunstancial }\end{array}$ & $\begin{array}{l}\text { excitación ante estímulos } \\
\text { adecuados }\end{array}$ \\
\hline $\begin{array}{l}\text { 10.Evidencia de psicopatología grave (psicosis) o ingreso } \\
\text { previo en psiquiátricos }\end{array}$ & $\begin{array}{l}\text { 5.Bajo control de la conducta } \\
\text { desviada }\end{array}$ \\
\hline 11.Acciones excéntricas, rituales, fantasías desviadas & 6.Carecer de pareja (.22) \\
\hline & 7. Perfil psicopático (Factor II de la \\
\hline 12. Presentar varias parafilias (abusos, violaciones) & Esala de Psicopatía de Hare, \\
\hline $\begin{array}{l}\text { 13.Perfil psicopático (Factor I de la Esala de Psicopatía } \\
\text { de Hare, PCL): Rasgos psicopáticos) (.18) }\end{array}$ & $\begin{array}{l}\text { PCL): Conducta antisocial, } \\
\text { salvo la que corresponde al } \\
\text { pasado) }\end{array}$ \\
\hline
\end{tabular}

Fuente: Elaboración propia a partir de diversos autores. Las correlaciones presentadas proceden de Quinsey et al. (1995)

Como puede verse en la tabla anterior, existe un amplio conjunto de factores estáticos de riesgo que, además de la menor edad y de factores de riesgo genéricos, condicionan específicamente la reincidencia de los agresores sexuales (con correlaciones conocidas de entre .13 y .22). Pese a todo, los factores dinámicos juegan también un papel decisivo en dicha reincidencia y constituyen, en todo caso, debido a su modificabilidad, los objetivos oportunos de los programas de tratamiento. Mientras que los factores estáticos se corresponden, en buena medida, con los elementos energizantes o motivadores de las agresiones (excitación, repertorios de conducta desviada, etc.), los factores dinámicos estarían formando parte de dos procesos decisivos en dichas agresiones (Farrington, 1996): 1) confiriendo direccionalidad antisocial al comportamiento, debido a la falta de habilidades para el logro de los objetivos deseados (entablar relaciones comunicativas y sexuales con personas adultas que consienten en dichas

Revista Española de Investigación Criminológica

Artículo 6, Número 4 (2006) $\quad$ www.criminología.net

ISSN: $1696-9219$ 
relaciones), y 2) facilitando los procesos desinhibitorios (creencias, distorsiones, falta de empatía, etc.) que precipitan la agresión.

\section{El tratamiento de los agresores sexuales mediante el programa "Control de la Agresión Sexual” (SAC), de Garrido y Beneyto (1996)}

En España el análisis específico de los delincuentes sexuales y la aplicación de programas con ellos se iniciaron en Cataluña en 1996, a partir de diversas investigaciones previas sobre la materia. La primera de estas investigaciones (Garrido, Redondo, Gil, Torres, Soler, y Beneyto, 1995) estudió una muestra de 29 violadores, autores de 226 delitos de diversas tipologías. La segunda (Garrido, Beneyto, y Gil, 1996) analizó a 33 delincuentes sexuales que habían abusado de menores y eran autores de 116 delitos. Una tercera investigación (Garrido, Gil, Forcadell, Martínez, y Vinuesa, 1998) estudió una muestra de delincuentes sexuales menores de edad con la finalidad de adaptar un programa específico para jóvenes.

Como resultado de ello y de acuerdo con las indicaciones generales del tratamiento cognitivo-conductual de los delincuentes (Beech y Mann, 2002; Brown, 2005; Budrionis y Jongsma, 2003; Garrido, 2005; Lipsey y Landerberger, 2006; Marshall y Serran, 2004; McGuire, 2001; Redondo, Sánchez-Meca y Garrido, 2002a, 2002b) se creó el primer programa específico para delincuentes sexuales adaptado al contexto español y denominado Programa de Control de la Agresión Sexual (SAC) (Garrido y Beneyto, 1996, 1997). La primera aplicación de este programa se llevó a cabo paralelamente en dos centros penitenciarios de la provincia de Barcelona: Quatre Camins y Brians (Roca y Montero, 2000). Este mismo programa, con algunas adaptaciones, se aplica en la actualidad en diversos centros penitenciarios españoles.

En este programa el tratamiento se concibe a partir de dos ejes complementarios (Navarro, 2004). En primer lugar, un eje de evaluación individual de cada sujeto, en el que se formulan hipótesis funcionales acerca de los factores que probablemente mantienen su agresión. En segundo lugar, un eje de intervención

Revista Española de Investigación Criminológica

Artículo 6, Número 4 (2006) $\quad$ www.criminología.net

ISSN: $1696-9219$ 
grupal a partir de todos aquellos conocimientos, habilidades, cambios cognitivos y emocionales que los agresores sexuales deben asimilar y modificar.

\subsection{La motivación para el tratamiento}

Se ha puesto de relieve una serie de correlatos clínicos que se asocian a la motivación de los agresores sexuales para participar en un tratamiento (Andrews y Bonta, 2003; Garrido et al., 1995; Groth, 1979; Quinsey et al., 1995; Maletzky, 1991; Marshall, 2001; Marshall y Barbaree, 1989), que se recogen en la tabla 2 a continuación:

Tabla 2. Correlatos clínicos favorables para el tratamiento

Desagrado con sus acciones delictivas y deseo de cambiar

Reconoce la existencia de factores relacionados con sus conductas de agresión sexual (fantasías, creencias, ira, alcohol)

Capaz de entablar relaciones afectivas

Capacidad verbal

Capacidad para aprender de la experiencia

No hubo violencia física en la comisión de los delitos

Capaz de controlar su conducta en alguna medida

Cierto ajuste en su pareja, trabajo y otras áreas sociales

No presenta psicosis ni otras deficiencias neurológicas graves

Como puede verse en la tabla anterior, algunos de dichos correlatos ("desagrado con sus acciones delictivas y deseo de cambiar", "reconoce la existencia de factores relacionados con sus conductas de agresión sexual", "capaz de controlar su conducta en alguna medida") hacen referencia a un importante factor de fondo: el grado de conciencia e incomodidad que tiene el sujeto acerca de sus conductas de agresión y, en consecuencia, el grado en que desea o intenta resolver el problema. Se trata, en suma, de la disposición para el cambio de conducta, algo que nos conecta en psicoterapia con la perspectiva de Prochaska y DiClemente acerca de los estadios de cambio (Prochaska y Diclemente, 1992; Prochaska y Prochaska, 1993). Según este modelo, explorado en múltiples problemas adictivos y psicopatológicos, para que el cambio de comportamiento sea

Revista Española de Investigación Criminológica

Artículo 6, Número 4 (2006) $\quad$ www.criminología.net

ISSN: $1696-9219$ 
viable (y, en consecuencia, para que el tratamiento sea más eficaz) es necesario que el sujeto se encuentre, como mínimo, en un estadio de comtemplación del problema, es decir que haya adquirido conciencia del mismo (que reconozca que tiene un problema) y se haya suscitado en él la inquietud de resolverlo pronto.

En el caso de los agresores sexuales, para que el individuo tenga "desagrado con sus acciones delictivas y deseo de cambiar", es necesario, para comenzar, que reconozca el delito, esto es que salga de los habituales procesos de negación que suelen acompañar a una conducta socialmente vergonzante y de fuerte rechazo como la agresión sexual.

A menudo los delincuentes sexuales tienden a percibirse a sí mismos como personas corrientes, incluso buenas personas, para lo cual minimizan y externalizan los hechos. Para ello, pueden automatizar una serie de procesos de pensamiento en cadena, en los que el sujeto utiliza mecanismos de justificación y se da a sí mismo mensajes como los siguientes (Navarro, 2004):

1. Sobreoptimismo: p.e., "podré resolverlo por mí mismo, sólo fue algo circunstancial”.

2. Resistencia al tratamiento: p.e., "no necesito ningún tratamiento, ya ha pasado mucho tiempo; además, cualquiera puede cometer un error; ya he pagado por ello".

3. Negación: p.e., "si no necesito tratamiento, ¿para qué pasar un mal trago explicando lo que pasó?"

4. Justificación: p.e., "a nadie le importa mi vida; si lo explico, seguro que lo contarán a personas que me importan y tendré que avergonzarme”.

5. Conclusión: p.e., "que lo explique no servirá para nada; no vale la pena".

El programa objeto de esta investigación requiere a sus participantes, inicialmente o tras un tiempo prudencial de aplicación, el reconocimiento del delito. Ello, además de facilitar el tratamiento, suele tener efectos emocionales beneficiosos para el sujeto. En el marco del tratamiento, el grupo representa en cierto sentido a la sociedad y es saludable que el sujeto pueda hablar de su delito con menor ansiedad y mayor objetividad, aprendiendo a convivir con lo que hizo, y

Revista Española de Investigación Criminológica

Artículo 6, Número 4 (2006) $\quad$ www.criminología.net

ISSN: $1696-9219$ 
experimentando, en suma, la aceptación del terapeuta y del grupo (Navarro, 2004). Este proceso requiere que el terapeuta estimule, dirija y valore los esfuerzos del sujeto en dirección al reconocimiento del delito, proceso en el que cada individuo tendrá su propio ritmo, sin que éste deba ser forzado.

\subsection{Objetivos y componentes del programa de tratamiento}

Este programa es de orientación cognitivo-conductual y en su fase de grupo tiene una duración promedio de 10-12 meses, a razón de 4 sesiones semanales de dos horas. El quinto día de la semana se dedica al seguimiento de cada individuo en entrevista individual y a cuestiones pendientes (revisiones de grado, permisos, etc.). Los objetivos terapéuticos generales del programa son los siguientes (Garrido y Beneyto, 1996):

1. Favorecer un análisis más realista por parte de los sujetos participantes en el mismo acerca de sus actividades delictivas.

2. Mejorar sus capacidades y habilidades de relación personal.

3. Mejorar sus posibilidades de reinserción y de no reincidir.

De modo más específico, el programa está estructurado en módulos o ingredientes que se dirigen a los déficit o factores de "necesidad criminogénica" más comunes de los delincuentes sexuales. Incorpora 6 ingredientes, cuyos fundamentos técnicos y objetivos terapéuticos se recogen sucintamente (en tabla 3) (Garrido y Beneyto, 1996; Garrido et al., 1995): 
Tabla 3. Ingredientes terapéuticos, fundamentos y objetivos del Programa de Control de la Agresión Sexual (SAC)

\begin{tabular}{|c|c|c|}
\hline $\begin{array}{l}\text { Módulos o } \\
\text { Ingredientes } \\
\text { terapéuticos }\end{array}$ & Fundamentos teóricos & Objetivos \\
\hline $\begin{array}{l}\text { 1.Distorsiones } \\
\text { cognitivas } \\
\text { (reestructuración } \\
\text { cognitiva) } \\
\text { (44 sesiones) }\end{array}$ & $\begin{array}{l}\text { Terapia Cognitiva de Beck y } \\
\text { otras aportaciones sobre } \\
\text { pensamientos automáticos y } \\
\text { erróneos y sobre } \\
\text { reestructuración }\end{array}$ & $\begin{array}{l}\text { Reestructurar la percepción y } \\
\text { distorsiones del sujeto sobre el } \\
\text { hecho delictivo, sus propios deseos y } \\
\text { su percepción de las mujeres, niños, } \\
\text { violencia, etc. }\end{array}$ \\
\hline $\begin{array}{l}\text { 2.Mecanismos de } \\
\text { defensa } \\
\text { (15 sesiones) }\end{array}$ & $\begin{array}{l}\text { Técnicas de neutralización de } \\
\text { Matza y Terapia de realidad } \\
\text { de Glasser }\end{array}$ & $\begin{array}{l}\text { Erradicar el uso de justificaciones y } \\
\text { promover la responsabilidad del } \\
\text { sujeto sobre su propia conducta }\end{array}$ \\
\hline $\begin{array}{l}\text { 3.Conciencia } \\
\text { emocional } \\
\text { (18 sesiones) }\end{array}$ & $\begin{array}{l}\text { Educación en autoexploración } \\
\text { emocional }\end{array}$ & $\begin{array}{l}\text { Aumentar la conciencia y el } \\
\text { repertorio emocional del sujeto }\end{array}$ \\
\hline $\begin{array}{l}\text { 4.Empatía } \\
\text { con la víctima } \\
\text { ( } 27 \text { sesiones) }\end{array}$ & $\begin{array}{l}\text { Trabajo de Marshall sobre } \\
\text { sensibilización hacia el dolor } \\
\text { y emociones ajenas; técnica } \\
\text { role taking de Platt et al. }\end{array}$ & $\begin{array}{l}\text { Enseñar al individuo a reconocer } \\
\text { emociones y sentimientos de otras } \\
\text { personas (víctimas), y aumentar su } \\
\text { capacidad de empatía }\end{array}$ \\
\hline $\begin{array}{l}\text { 5.Prevención } \\
\text { de recaídas } \\
\text { (17 sesiones) }\end{array}$ & $\begin{array}{l}\text { Pithers y Laws a partir de } \\
\text { Marlat y } \text { Gordon (en } \\
\text { alcohólicos) }\end{array}$ & $\begin{array}{l}\text { Mejorar la capacidad del sujeto para } \\
\text { anticipar y romper las cadenas de } \\
\text { precursores (cognitivos, fisiológicos, } \\
\text { emocionales, conductuales y } \\
\text { ambientales) de sus delitos }\end{array}$ \\
\hline $\begin{array}{l}\text { 6.Estilo de vida } \\
\text { positivo } \\
\text { (17 sesiones) } \\
\end{array}$ & $\begin{array}{lcc}\text { Técnicas } & \text { psicoeducativas } & \text { y } \\
\text { modelado } & \text { estructurado de } \\
\text { Goldstein } & & \\
\end{array}$ & $\begin{array}{l}\text { Mejorar sus habilidades de } \\
\text { planificación de vida y sus hábitos y } \\
\text { rutinas cotidianas }\end{array}$ \\
\hline
\end{tabular}

\subsection{Equipo de tratamiento}

El equipo de tratamiento responsable de este programa está formado por psicólogos, juristas criminólogos, educadores y trabajadores sociales. Los terapeutas principales del programa de agresores sexuales son los psicólogos, que efectúan tanto la parte central de la evaluación inicial como la aplicación de las técnicas de tratamiento recogidas en la tabla 3. 


\subsection{Formatos y contextos de aplicación del programa}

El programa se desarrolla en tres formatos y niveles de intervención:

1. Nivel individual (1 día por semana): para la realización de la evaluación inicial de los sujetos y, a lo largo del desarrollo del programa, para el seguimiento y motivación de cada sujeto, posibles incidencias y aplicación de pruebas psicológicas.

2. Nivel grupal (cuatro días por semana): es el núcleo principal de la intervención, en que se trabaja mediante los módulos psicosociales del programa.

3. Intervención y seguimiento en el exterior: una vez que el sujeto accede nuevamente a la comunidad, primero de modo supervisado (salidas programadas, en grupo o individuales, con acompañamiento de un terapeuta), restringido (permisos de salida de unos días, régimen abierto) o autónomo (libertad condicional y libertad definitiva). En estos seguimientos participan trabajadores sociales y los propios terapeutas del programa, que realizan mensualmente una sesión de seguimiento en el exterior de la prisión, en la que participan tanto liberados condicionales como internos que pueden salir de modo supervisado.

\subsection{Selección de los grupos de tratamiento y evaluación inicial de los sujetos}

Los grupos tratados en este programa se componen de un número de sujetos que oscila entre 10 y 15 , seleccionados entre los internos del centro condenados por delitos sexuales que cumplan los dos siguientes requisitos (Navarro 2004): 1) que estén en condiciones jurídico-penales de obtener la libertad condicional en el plazo de 3 a 4 años (es decir, que al inicio de programa estén próximos al cumplimiento de las tres cuartas partes de la condena de prisión que cumplen), y 2) que tengan un reconocimiento, aunque sea mínimo o parcial, del delito y una cierta motivación inicial para participar en el programa. Con el propósito de aumentar y mantener dicha motivación puede pactarse y suscribirse entre cada interno y el terapeuta director del grupo un acuerdo conductual. En éste el sujeto se compromete a asistir y participar en las sesiones diarias del programa y

Revista Española de Investigación Criminológica

Artículo 6, Número 4 (2006) $\quad$ www.criminología.net

ISSN: $1696-9219$ 
el terapeuta, por su parte, se compromete a valorar positivamente dicha participación y esfuerzo en las futuras propuestas de beneficios penitenciarios (posibles permisos, libertad condicional y régimen abierto).

Se efectúa una evaluación inicial mediante entrevista semiestructurada y la aplicación de la escala de psicopatía PCL-SV de Hare y de una escala de riesgo de 10 ítems diseñada al efecto (Navarro, 2004).

Se incluye como máximo un sujeto con perfil psicopático por grupo (evaluación PCL-SV de Hare, con una puntuación comprendida entre 18 y 24). La razón para adoptar esta medida estriba en facilitar, de este modo, un mejor control por el terapeuta de los posibles intentos de manipulación que puedan efectuar personas con alto nivel de psicopatía, a la vez que al resto del grupo le resulta también más fácil reconocer dichos intentos.

En síntesis, se espera que todas las intervenciones mencionadas (integrantes del Programa de Control de la Agresión Sexual) se traduzcan en mejoras conductuales, cognitivas y emocionales consistentes con los objetivos mencionados y, a la postre, en una reducción del riesgo de cometer nuevos delitos o de la frecuencia y gravedad de los mismos.

\section{3. ¿Sirve este tratamiento para rehabilitar a los delincuentes sexuales?}

El "Programa de Control de la Agresión Sexual" se viene aplicando en el Centro Penitenciario Brians desde 1996 hasta la actualidad. Algunos sujetos que fueron tratados ya han cumplido sus condenas de prisión y han salido en libertad. Ello ha permitido desarrollar la evaluación que aquí se presenta, para conocer si el tratamiento logra o no rehabilitar a los delincuentes que fueron tratados.

\subsection{Objetivos e hipótesis de este estudio}

Así pues, el objetivo fundamental de esta investigación es evaluar la eficacia del tratamiento descrito de los agresores sexuales, una vez que ha sido 
tratado un número amplio de sujetos y que ha transcurrido el tiempo suficiente para poder efectuar un seguimiento razonable de su comportamiento en la sociedad.

La hipótesis principal de partida es la siguiente: los sujetos que han completado el tratamiento (Grupo de tratamiento) obtendrán resultados significativamente mejores en las variables dependientes evaluadas (menor reincidencia sexual, no sexual y total, y menor gravedad de los delitos que puedan cometer) que los sujetos que no han recibido tratamiento (Grupo de control).

Esta primera hipótesis se matiza con una segunda hipótesis complementaria: como muestra la bibliografía especializada (Rice, Harris y Quinsey, 2001) los efectos positivos esperables del tratamiento, que por definición opera sobre la modificación de factores dinámicos de riesgo (hábitos y valores delictivos, distorsiones y justificaciones, falta de empatía, estilo de vida desestructurado, etc.), serán modulados (y probablemente reducidos) a partir de variables estáticas de riesgo, tales como el historial delictivo previo del sujeto, la menor edad, la versatilidad delictiva, el tipo de víctimas a las que agrede o su posible perfil psicopático. Todos estos factores estáticos son inherentes al sujeto o a su pasado, por lo que, aunque tienen un efecto potenciador del riesgo de reincidencia, no pueden ser alterados por la intervención terapéutica. Así pues, se espera encontrar que la eficacia del tratamiento no sea unívoca, sino mayor o menor en función de la menor o mayor presencia en cada sujeto de factores de riesgo estáticos (inalterables).

\subsection{Muestras evaluadas y diseño de investigación}

La población total de agresores sexuales que han cumplido condena en el Centro Penitenciario Brians, desde la inauguración de este centro en mayo de 1991 hasta el 31 de diciembre de 2002, es de 346 sujetos, que en conjunto son autores de más de 770 delitos sexuales (con un promedio de 2,23 delitos sexuales por sujeto). También son responsables de más de 630 delitos no sexuales. De esta población total se han extraído y seleccionado los grupos objeto de esta investigación: uno tratado $(n=49)$ y otro control $(n=74)$, de los que se ha efectuado un seguimiento (una vez liberados y reintegrados a la comunidad) a lo largo de un periodo promedio de 3 años y 8 meses.

Revista Española de Investigación Criminológica Artículo 6, Número 4 (2006) $\quad$ www.criminología.net 
Esta investigación es un estudio retrospectivo realizado mediante un diseño de grupo de control no equivalente, es decir no seleccionado al azar. No obstante se han tomado diversas medidas de depuración metodológica para erradicar los sesgos más importantes que podrían amenazar la homogeneidad y equivalencia de los grupos. Como resultado de ello, los grupos pueden considerarse equivalentes.

Para la recogida de los datos se ha creado una plantilla de codificación de variables, que incorpora una estructura de cuatro grandes categorías: A) variable independiente (aplicación vs. no aplicación del tratamiento), B) variables moduladoras (demográficas, de carrera criminal, víctimas, clínicas, etc.), y C) variables criterio o dependientes (distintos parámetros de reincidencia). En total se han analizado 51 variables. 


\subsection{Resultados}

¿Es eficaz el tratamiento?

Los resultados más importantes de esta investigación, en relación con su principal objetivo son los siguientes (véase la Figura 1): de los 49 sujetos integrantes del grupo de tratamiento han reincidido en delitos sexuales, a lo largo de un periodo de seguimiento de cuatro años, 2 sujetos $(4,1 \%)$ y en delitos no sexuales 1 sujeto $(2,0 \%)$, lo que suma una reincidencia total de 3 sujetos $(6,1 \%)$. Frente a ello, de los 74 integrantes del grupo de control, durante un periodo de seguimiento de tres años y medio, 13 sujetos (el 18,2\%) han reincidido en delitos sexuales y 10 más en delitos no sexuales (13,6\%), lo que aúna un total de 23 sujetos reincidentes (el 31,8\%). Todas estas diferencias entre los grupos resultan estadísticamente significativas.

Estos resultados confirman en esencia la hipótesis principal de esta investigación, a saber: la eficacia del tratamiento cognitivo-conductual aplicado con los agresores sexuales en la prisión de Brians. El tratamiento logra reducir la reincidencia sexual en un $14,1 \%$, que resulta de la diferencia entre la tasa del grupo de control, que es de 18,2\%, y la del grupo de tratamiento, que es del 4,1\%. 


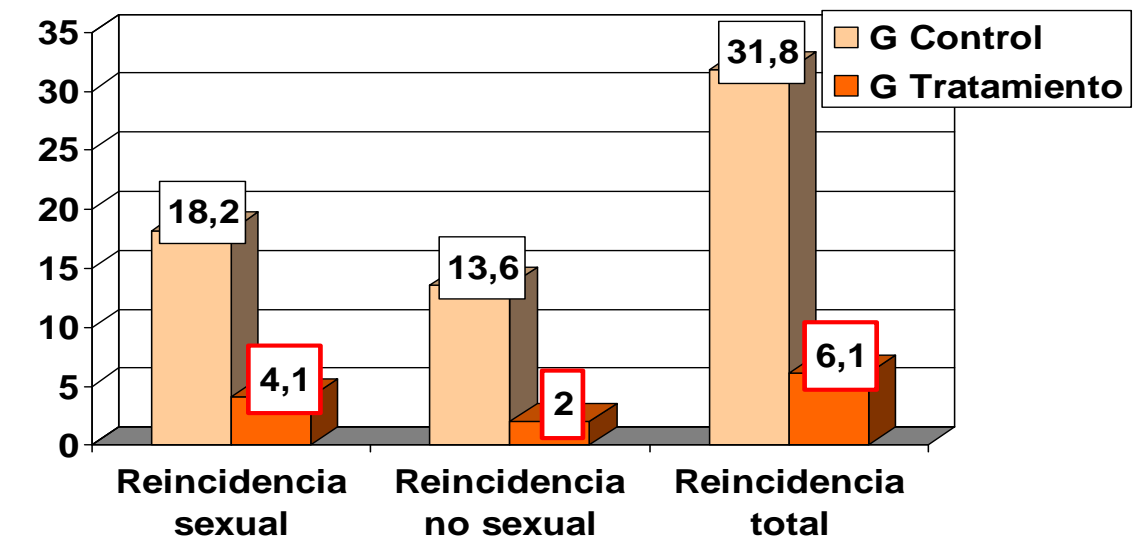

Figura 1. Reincidencia sexual, no sexual y total del Grupo de Control y del Grupo de Tratamiento

¿Qué variables modulan la reincidencia sexual?

Se han explorado también las posibles interacciones entre el resultado reincidencia/no-reincidencia sexual y las diversas variables moduladoras consideradas, como posibles factores de riesgo, en el estudio. Del análisis bivariado de la reincidencia sexual (para una muestra total de 123 sujetos, suma de los 49 tratados más los 74 controles), se encontró capacidad explicativa de la reincidencia sexual en 26 variables o factores de riesgo.

Los datos obtenidos confirman en buena medida la segunda hipótesis formulada: al analizar las características de los sujetos reincidentes (con independencia de que hayan sido tratados o no) aparecen interacciones con factores de riesgo fundamentalmente estáticos o inmodificables (menor edad al salir en libertad, carreras criminales más prolongadas, mayor número de delitos, víctimas desconocidas, etc.). Así, los sujetos reincidentes, además de haber recibido tratamiento en menor proporción (14,3\%), presentan riesgos estáticos diversos que, sin duda, están jugando un papel decisivo en la prolongación de sus carreras criminales ( $\sin$ o con tratamiento). 


\section{Conclusiones}

En la figura 2 se representa la distribución de la reincidencia en las muestras de agresores sexuales no tratados: de acuerdo con la investigación internacional alrededor del $80 \%$ no reinciden, mientras que acaban reincidiendo en torno a un 20\% (Lösel, 2002; Quinsey et al., 1995). ¿Qué sucede, si se aplica un tratamiento como el aquí presentado? Lo que sucede es que en general el tratamiento puede rebajar en 5\%-10\% la tasa de reincidentes esperables (20\%), es decir puede reducirla a la mitad (Beech y Mann, 2002; Hall, 1995; Redondo y Sánchez-Meca, 2003). (Esta reducción de la reincidencia es proporcional a los beneficios del tratamiento en otras tipologías de delincuentes: véase una revisión en Welsh y Farrington, 2006). Pese a todo, resta un porcentaje residual de 10\%-15\%, de sujetos de alto riesgo, que probablemente van a acabar reincidiendo a pesar de haber participado en el tratamiento. Estos sujetos suelen corresponderse con aquellos que presentan el peor pronóstico en factores estáticos de riesgo, tales como la menor edad y el inicio temprano en la delincuencia.

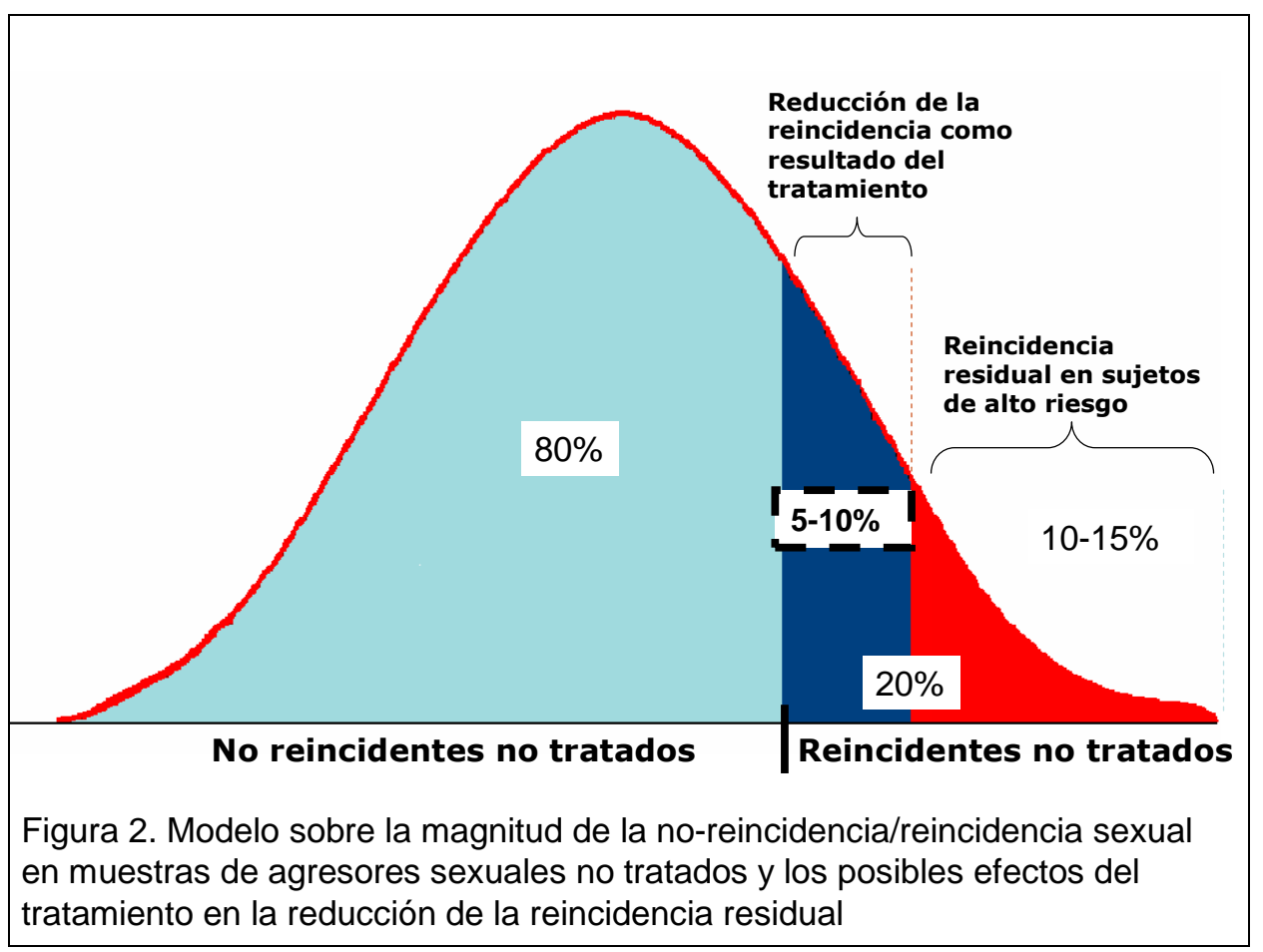

Revista Española de Investigación Criminológica

Artículo 6, Número 4 (2006) $\quad$ www.criminología.net

ISSN: $1696-9219$ 
El modelo de la figura 2 es teórico, y representa lo que los estudios evaluativos suelen encontrar, sin tratamiento y cuando se aplica tratamiento. Los datos obtenidos en este estudio son, en principio, algo más optimistas, ya que el ahorro en reincidencia sexual, como resultado del tratamiento, es del 14,1\% (sobre una reincidencia base del grupo control del 18,2\%). Así pues, resta una reincidencia residual (sujetos tratados que, pese a todo, reinciden) del $4,1 \%$.

En conclusión, los resultados obtenidos sugieren una considerable potencia rehabilitadora del tratamiento cognitivo-conductual aplicado en la prisión de Brians (Barcelona, España). Este estudio claramente prueba, en términos científicos, que la aplicación del tratamiento reduce la tasa de reincidencia de los agresores sexuales tratados, desde una tasa que sería esperable del $18 \%$ a una del $4 \%$, para un periodo de seguimiento promedio de casi cuatro años. No obstante, debido a la prolongada potencialidad delictiva de los agresores sexuales, estos resultados iniciales sólo constituyen una primera aproximación evaluativa que deberá ser completada mediante un seguimiento más prolongado de los sujetos que permita evaluar su reincidencia a más largo plazo.

Aunque en este estudio no se han podido evaluar otras variables y factores de protección, es muy probable que, además del tratamiento psicológico aplicado, los sujetos tratados hayan contado con otros factores favorables para su reinserción social, tales como la ayuda de sus familias y unas condiciones laborales y sociales favorables. Casi con toda seguridad el tratamiento aplicado ha promovido cambios personales en los sujetos (en sus habilidades, actitudes, modos de pensamiento y controles emocionales) que han contribuido a la inhibición de la agresión sexual. Sin embargo, estos cambios y mejoras personales también han tenido que contar, para hacerse realidad, con contextos favorables en los que pudieran manifestarse. La evaluación combinada de cambios personales y factores sociales de reinserción constituye un reto de la máxima importancia para la investigación futura en la rehabilitación tanto de los delincuentes sexuales en particular como de los delincuentes en general. 


\section{Referencias}

Andrews, D., y Bonta, J. (2003). The Psychology of Criminal Conduct ( $3^{\mathrm{a}}$ ed.). Cincinnati (EEUU): Anderson Publishing Co.

Beech, A., y Mann, R. (2002). Recent developments in the assessment and treatment of sexual offenders. En J. McGuire, Offender Rehabilitation and Treatment (pp. 259-288). Chichester: John Wiley \& Sons.

Berlin, F.S. (2000). The Etiology and Treatment of Sexual Offending. En D. Fishbein: The Science, Treatment, and Prevention of Antisocial Behaviors (cap. 21). Kingston, New Jersey: Civic Research Institute.

Brown, S. (2005). Treating Sex Offenders. Cullompton, Devon (UK): Willan Publishing.

Budrionis, R., y Jongsma, A.E. (2003). The Sexual Abuse Victim and Sexual Offender Treatment Planner. Hoboken (New Jersey): John Wiley \& Sons.

Echeburúa, E., y Guerricaechevarría, C. (2000). Abuso sexual en la infancia: víctimas y agresores. Un enfoque clínico. Barcelona: Ariel.

Farrington, D.P. (1996). The explanation and prevention of youthful offending. En P. Cordelia y L. Siegel (eds.) Readings in contemporary criminological theory. Boston: Northeastern University Press.

Gallagher, C. A., Wilson, D. B., Hirschfield, P., Coggeshall, M. B., y McKenzie, D. L. (1999). A quantitative review of the effects of sex offender treatment on sexual reoffending. Corrections Management Quarterly, 3, 19-29.

Garrido, V. (2005). Qué es la psicología criminológica. Madrid: Biblioteca Nueva.

Garrido, V., y Beneyto, M.J. (1996). El control de la agresión sexual. Un programa de tratamiento para delincuentes sexuales en prisión y en la comunidad. Valencia: Ed. Cristobal Serrano Villalba.

Garrido, V., y Beneyto, M.J. (1997). La valoración psicológica de los agresores sexuales: los delitos, la reincidencia y el tratamiento. En Cuadernos de Derecho Judicial:Delitos contra la libertad sexual. Escuela Judicial. Consejo General del Poder Judicial.

Garrido, V., Beneyto., M.J., y Gil, A. (1996). Els delinqüents sexuals institucionalitzats. Un estudi del agressors de nens. Barcelona: Centre d'Estudis Jurídics i Formació Especialitzada. 
Garrido, V., Gil, A., Forcadell, A., Martínez, R.M., y Vinuesa, M.R. (1998). Joves agressors sexuals: avaluació, diagnòstic $i$ tractament. Memoria de investigación. Barcelona: Centre d'Estudis Jurídics i Formació Especialitzada. Generalitat de Catalunya.

Garrido, V., Redondo, S., Gil., A., Torres, G., Soler, C., y Beneyto, M.J. (1995). Delinqüens sexuals en les presons. Barcelona: Generalitat de Catalunya.

Gendreau, P., Little, T., y Goggin, C. (1996). A meta-analysis of the predictors of adult offender recidivism: What works? Criminology, 34(4), 575-607.

Greenberg, D.M., y Bradford, J.M.W. (1997). Treatment of the paraphilic disorders: A review of the role of the selective serotonin reuptake inhibitors. Sexual Abuse: A Journal of Research and Treatment, 9, 349-360.

Groth, A.N. (1979). Men who rape: The psychology of the offender. New York: Plenum Press.

Hall, N. G. C. (1995). Sexual offender recidivism revisited: A meta-analysis of recent treatment studies. Journal of Consulting \& Clinical Psychology, 63, 802809.

Lipsey, M.W., y Landerberger, N.A. (2006). Cognitive-Behavioral Interventions. En B.C. Welsh y D.P. Farrington, Preventing Crime: What Works for Children, Offenders, Victims and Places (pp. 57-71). Dordrencht, The Netherlands: Springer

Lösel, F. (2002). ¿Sirve el tratamiento para reducir la reincidencia de los delincuentes sexuales?. En S. Redondo, Delincuencia sexual y sociedad (págs. 361-396). Barcelona: Ariel.

Maletzky, B.M. (1991). Treating the sexual offender. London: Sage.

Marshall, W.L., y Barbaree, H.E. (1989). Sexual violence. En K. Howells y C.R. Hollin (Eds.), Clinical approaches to violence. Chichester: John Wiley and Sons. Marshall, W.L., y Barbaree, H.E. (1990). An integrated theory or the etiology of sexual offending. En W.L. Marshall, D.R. Laws y H.E. Barbaree (Eds.), Handbook of sexual assault: Issues, theories, and treatment of the offender (págs. 257-275). New York: Plenum Press.

Marshall, W.L. y Redondo, S. (2002). Control y tratamiento de la agresión sexual. En S.Redondo (Coord.), Delincuencia sexual y sociedad (págs. 301-328). Barcelona: Ariel.

Revista Española de Investigación Criminológica Artículo 6, Número 4 (2006) $\quad$ www.criminología.net

ISSN: $1696-9219$ 
Marshall, W.L. y Serran, G.A. (2004). Tratamiento del agresor sexual. En J. Sanmartín (Coord.), El laberinto de la violencia (pp. 309-319). Barcelona: Ariel.

McGuire, J. (2001). What works in correctional intervention? Evidence and practical implications. En G.A.Bernfeld, D.P. Farrington, y A.W. Leschied, Offender Rehabilitation in Practice (pp. 25-43). Chichester (UK): Wiley \& Sons. Navarro, J.C. (2004). El tratamiento de los agresores sexuales en prisión. I Congreso de Psicología Jurídica y Forense en Red, 3-14 de Mayo de 2004.

Prentky, R.A. (2003). Remediation of coercive sexual behavior. En B.K. Schwartz, Correctional Psychology: Practice, Programming, and Administration (pp. 13-1 to 13-33). Kinston (New Jersey): Civic Research Institute, Inc.

Prochaska, J.O. y DiClemente, C.C. (1992). Stages of change in the modification of problem behaviors. En M. Hersen, R.M. Eisler y P.M. Miller (Eds.), Progress in behavior modification (págs. 184-214). Sycamore, IL: Sycamore Press.

Prochaska, J.O. y Prochaska, J.M. (1993). Modelo transteórico de cambio para conductas adictivas. En M. Casas y M. Gossop (Eds.), Tratamientos psicológicos en drogodependencias: recaída y prevención de recaídas (págs. 85-136). Barcelona: Ediciones Neurociencias.

Quinsey, V.L., Lalumière, M.L., Rice, M.E., y Harris, G.T. (1995). Predicting sexual offenses. En J.C. Campbell (Ed.), Assessing dangerousness: Violence by sex offenders, batterers, and child abusers (pp. 114-137). Thousand Oaks, CA: Sage.

Redondo, S. (2002). Delincuencia sexual: mitos y realidades. En S. Redondo (coord.), Delincuencia y sociedad (Cap. 1, págs. 35-52). Barcelona: Ariel.

Redondo, S. y Sánchez Meca, J. (2003). Guía de tratamientos psicológicos eficaces para la delincuencia juvenil. En M. Pérez, J.R. Fernández Hermida, C. Fernández Rodríguez, e I. Amigo Vázquez, Guía de tratamientos psicológicos eficaces III. Infancia y adolescencia (págs. 183-214). Madrid: Pirámide.

Redondo, S., Sánchez-Meca, J., y Garrido, V. (2002a). Crime treatment in europe: a review of outcome studies. En J. McGuire, Offender Rehabilitation and Treatment (pp. 113-141). Chichester: John Wiley \& Sons.

Redondo, S., Sánchez-Meca, J. y Garrido, V. (2002b). Los programas psicológicos con delincuentes y su efectividad: La situación europea. Psicothema, 14 (Supl.), 164-173.

Revista Española de Investigación Criminológica

Artículo 6, Número 4 (2006) $\quad$ www.criminología.net

ISSN: $1696-9219$ 
Rice, M.E., Harris, G.T., y Quinsey, V.L. (2001). Research on the treatment of adult sex offenders. En J.B. Ashford, B.D. Sales, y W.H. Reid, Treating Adult and Juvenile Offenders With Special Needs (pp. 291-312). Washington, D.C.: American Psychological Association.

Roca, M., y Montero, P. (2000). Intervención con psicópatas en prisión. En A. Raine y J. Sanmartín: Violencia y psicopatía. (Estudios sobre violencia, $\mathrm{n}^{\mathbf{o}} 4$ ). Barcelona: Ariel/Centro Reina Sofía para el Estudio de la Violencia.

Rösler, A., y Witztum, E. (2000). Pharmacotherapy of Paraphilias in the Next Millennium. Behavioral Sciences and the Law, 18, 43-56.

Vandiver, D.M. (2006). A prospective analysis of juvenile male sex offenders: Characteristics and Recidivism rates as adults. Journal of Interpersonal Violence, 21(5), 673-688.

Welsh, B.C. y Farrington, D.P. (2006). Conclusions and Directions from EvidenceBased Crime Prevention. En B.C. Welsh y D.P. Farrington, Preventing Crime: What Works for Children, Offenders, Victims and Places (pp. 227-237). Dordrencht, The Netherlands: Springer. 